\title{
Data Acquisition and Processing Samples of an Explosive Material using Arm Microcontroller (ARM9263)
}

\author{
Gosukonda \\ Subrahmanyam \\ M.Tech (Embedded Systems), \\ Gudlavalleru Engineering \\ College, \\ Gudlavalleru,krishna(Dist.), \\ Andhra Pradesh
}

\author{
Jaya Prakash \\ M.Tech, \\ Senior Manager, \\ Telecom dept(TCD), \\ E.C.I.L, Hyderabad, \\ Andhra Pradesh
}

\author{
E.V.Vijay \\ Asst.Proffessor, \\ Gudlavalleru Engineering \\ College, \\ Gudlavalleru, Krishna (Dist.), \\ Andhra Pradesh
}

\begin{abstract}
The main aim of the project is to design and Development of data acquisition card and Process parameters of explosive materials in ARM Microcontroller to capture, store and analyze the data and Display characteristic waveform on LCD Screen. The data Acquisition card integrated into ARM9263 processor board. This can be widely used in Defense and army to detect the bombs and RDXs and to protect soldiers and people from harmful substances and to take remedy actions from explosives. The software coding of the total project is realized by using EMBEDDED $\mathrm{C}$ language in the KEIL software and the total hardware is realized by using $\mathrm{DAC}$ card, ADC with the processor.
\end{abstract}

\section{Keywords}

ARM9263 Embedded C, Keil, ADC

\section{INTRODUCTION}

Data acquisition is the process of sampling the signals that to measure real world physical conditions and converting the resulting samples into digital numeric values. These values can be manipulated by the computer. Data acquisition systems typically convert the analog waveforms into digital values for their processing. The components of data acquisition systems include:

- The Sensors that they will convert the physical parameters to the electrical signals.

- A Signal conditioning circuitry to convert the sensor signals into a form that can be converting to the digital values.

- Analog-to-digital converters, which convert the conditioned sensor signals to digital values

The Data acquisition and processing samples of explosives represents the detection of explosive materials like TNT, RDX, NACRO etc....it is generally called as Ion-Mobility Spectrometer .It is used to detect the explosives based on the drift velocity of the generated ions. Drift tube generates the ions from the samples placed. Based on the drift velocity of the ions, generated current will vary. As Drift tube output is connected to pre-amplifier, the drift tube output voltage in turn depends on the drift velocity of the sample. The pre amplifier output is connected to the ADC board (THS1206), which is integrated with the ARM board (AT91SAM9263). ADC converts the continuous analog signal to discrete Digital values.

DAPS(Data Acquisition and processing samples) captures the output voltage of the pre amplifier using ADC, based on the peak value of the collected ADC sample data, drift time is computed. Drift Time taken by the sample is compared with the available library data. If the drift time taken by the sample matches with any of the drift time values specified in the library, alarm will be rung and the name of the explosive detected is displayed along with the Characteristics like ADC peak voltage value (in volts) \& time (in milliseconds). The Total unit kept in a big chamber and which is having high voltage power supply.

In the project mainly describes how the explosive materials are detected and processed . The total project which contain different hardware units via...DAC, ADC, ARM9, LCD display.

\section{PROPOSED METHOD OF DATA ACQUISITION AND PROCESSING SAMPLES OF AN EXPLOSIVE MATERIAL BY USING ARM9}

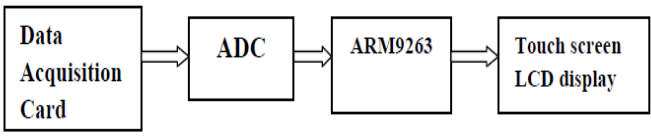

Fig.1 Block Diagram of D.A.P.S

In the proposed method the entire samples will be going too processed in drift tube. The explosive samples will be kept in the container and then going too processed by the data acquisition unit and to be display on touch screen LCD display.

Data acquisition systems, as the name refer to products and processes used to collect information. In the simplest form, a technician logging the temperature of an oven on a piece of paper is performing data acquisition.

Technology has increased, this type of process has simplified and made more accurate, useful and reliable through electronic equipment. Data acquisition products serve as an important point in a system. Data Acquisition system tying together a wide variety of products, such as sensors is used to indicate temperature, flow level and pressure.

Communication is based on the data acquisition products, those products are interfaced with a computer through communication port. The most widely used communication interface for the short distances is RS-232. In RS-232 the communication is serial communication for one device to one 
computer communication port. The speeds of the RS232 up to $115 \mathrm{~K}$ baud (bits per second)

Mostly 7 or 8 bits are transmitted to represent a character or a digit. The ASCII code provided a standard definition. The ASCII code will allow the alphanumeric characters to be represented as a string of bits. Other serial communication interfacesIncludesRS-422 andRS-485.

RS422 and 485 both will have the ability to communicate longer distances with the multiple units. Two common parallel communication interfaces are the Centroid and IEEE488.

Parallel interfaces communicate data At 8 or more bits at single time. The Centronic interface is the one common parallel Interface used to connect printers to a computer. The IEEE-488 interface provides a high speed parallel interface for Up to 15 devices.

An analog to digital Converter is a device that can convert the continuous physical quantity to a digital number that represents the quantity's amplitude. The conversion involves about quantizing of the input. It is necessary to introduce a small amount of error. An ADC performs the conversions ("samples" the input) periodically. The result will be the sequence of digital values that have converted a continuoustime and continuous-amplitude analog signal to a discretetime and the discrete-amplitude digital signal. An ADC is defined by its bandwidth and its signal to noise.

The AT91SAM9263 is a 32-bit microcontroller. It is based on the ARM926-EJ-S processor.

Touch screen system generally consists of the two parts. The touch detection devices and the touch screen controller. Touch detection device is attached in front of the monitor screen, for detecting a user touch position. The touch screen controller's main role is the touch point detecting means to receive touch information and convert it into contact coordinates. It also can receive the commands sent to the $\mathrm{CPU}$ and executed.

With advances in technology, touch-screen technology also experienced a gradual upgrade from low to high and the development process. According to working principle, touch screen LCDs generally divided into four categories they are resistive touch screen and capacitive touch screen. The others are infrared touch screen and the surface acoustic wave touch screen.

\subsection{Ion Mobility Spectrometer Data Acquisition \& Display Unit}

Ion Mobility spectrometry display unit captures analyses and displays the wave and characteristics of explosive sample introduced in the Drift tube sampler unit.

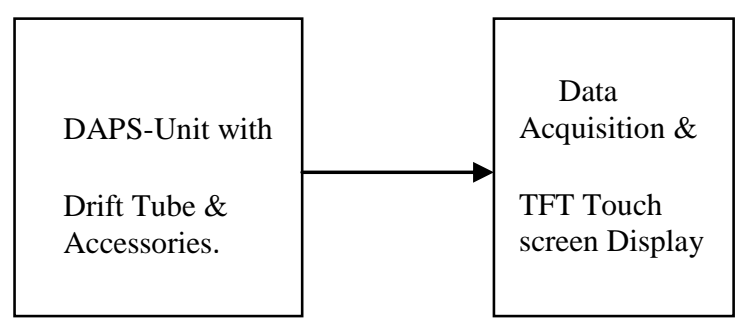

Fig.2 Block diagram of DAPS Unit with Drift tube

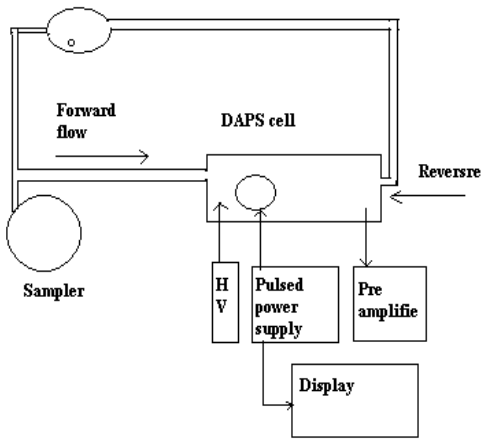

Fig.3 Data Acquisition External Layout

The ions propagate through drift tube in which the ion particles are collected by the collector and then amplified by the amplifier. How much time the particle will take to reach the collector from the amplifier is called "drift velocity". The total unit is connected to the DAC card and then processed.

D.A.P.S unit which contain drift tube and drift tube which allows the electrons to propagating through it. The electrons will travel very fast in the free space of drift tube and then processed by DAC card.

\subsection{Data Acquisition Card}

The Data Acquisition Card consists of

1. Level shifter.

2. Amplifier.

3. Timer circuit.

4. Clippers

5. Clampers

The entire designing is going to done by using ORCAD9 tool. In the entire design part net lists are going to be taken. By using the net lists footprints are going to be taken.

All the circuits are going to be designed by using footprints and the each unit fabricated on the single board.

\section{SIMULATION RESULTS}

These are results taken from DSO. The same wave is displayed on LCD display.

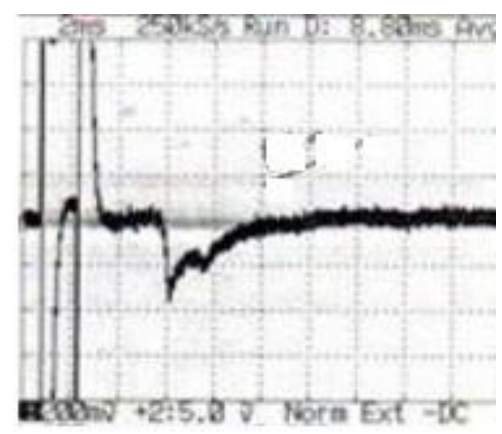

Fig .4Background (without sample) 


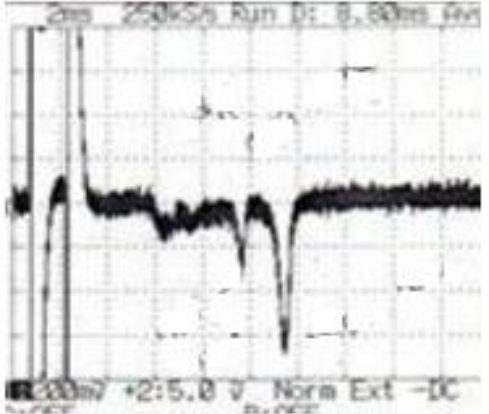

Fig 5. Wave with TNT \& RDX introduced

The DAPS cell is tested for negative ions detection. The sample RDX \& TNT are introduced into the sampler, as individual sample and combine sample. In both the case expected result is reported.

The first requirement of a DAPS system is a suitable ion source. The source is typically a beta emitter. In a dry air system the ionic species generated are pre-dominantly $\mathrm{N}+2$ or $\mathrm{O}+2$ Positive ions and $\mathrm{O}-2$ negative ions. These ions are referred as reactant ions. When molecules of the sample species are introduced into the ion source region these molecules will be interacted with the reactant ions. A charge transfer will take place creating ions of the sample species. TNT will undergo charge transfer reactions,

$\mathrm{TNT}+\mathrm{A} \rightarrow \mathrm{TNT} \pm \mathrm{A}$,

RDX will give the ion-molecule reactions with the reactant ions,

$$
\mathrm{RDX}+\mathrm{A}-\rightarrow(\mathrm{RDX} \pm \mathrm{A})
$$

The reduced ion mobility times correspond to the molecular weights of the addition compounds [RDX-O(H2O) ]-, [RDXO2]-. Therefore, the resultant IMS plasma gram of RDX contains 4distinct peaks. These ionic species will move toward the ion gate under the influence of the electric field. The polarity of the latter controls, Explosive substances form negative ions, most of the narcotics, like heroin and cocaine, form positive ions.

In the drift region the sample ions move under the influence of the applied electric field. Due to the collision between the sample ions and the drift gas molecules, the separation will be going depending on the mobility of the sample ions. Ions with a higher mobility will travel the length of the drift region in a shorter time .The mobility of the ions will depend on their size, mass and shape of the ions.

For the each opening of the electronic gate, a pulse of the electronic charge representing different ionic species in the sample will arrive at the ion collector separated in time. The spectrum of the ion current is referred to as a plasma gram.

\section{CONCLUSION}

This method is new approach to Processing samples of an explosive material. It is very simple when we are using ARM controller. By using these techniques we will easily detect the explosive samples. It is also less cost. It will detect the explosives with in less span of time $(<5 \mathrm{sec})$.

\section{REFERENCES}

[1] G.A. Eiceman, Z. Karpas, Ion Mobility Spectrometry, vol 31,2nd edition. 2005

[2] Alexandra. Shvartsburg, Differential Ion Mobility Spectrometry: Nonlinear Ion Transport and Fundamentals of FAImS, 2nd edition, Boca Raton, FL., USA: CRC Press. ISBN 9781420051063, vol.34, 2008.

[3] Charles L. Wilkins, Sarah Trimpin, ed. Ion Mobility Spectrometry - Mass Spectrometry: Theory and Applications. Boca Raton, FL., USA: CRC Press, vol.22, 2008.

[4] Kanu AB, Dwivedi P, Tam M, Matz L, Hill HH. "Ion mobility-mass spectrometry". J Mass Spectro, vol. 43 (1): 1-22, January 2008.

[5] Zolotov, “IonMobilitySpectrometry.” Journal of Analytical Chemistry, vol. 61 (6),2006.

[6] T.R. Hogness und R.W. Harkness, Phys.Rev. 32(1928) 936.

[7] M.M. Mann, R. Hustrulid und J.T. Tate, Phys.Rev. 58 (1940) 340 .

[8] E.A. Mason, H.W. Schamp, Jr., Ann. Phys. (N.Y.)4 (1958) 233.

[9] W.E. McDaniel, Collisional Phenomena in IonizedGases, John Wiley\& Sons, New York, 1964.

[10] D.L. Albriton und E.W. McDaniel, J. Phys. Chem.171 (1968) 94.

[11] M.M. Metro und R.A. Keller, J. Chromatogr. Sci12 (1974) 673. 